\title{
Editorial: Special Issue on Computing and Combinatorics
}

\author{
Donglei $\mathrm{Du}^{1} \cdot$ Dachuan $\mathrm{Xu}^{2}$
}

Published online: 26 February 2018

(C) Springer Science+Business Media, LLC, part of Springer Nature 2018

This special issue is associated with the 21st Annual International Computing and Combinatorics Conference (COCOON) in Beijing, China, August 4-6, 2015.

Papers for this special issue were originated from participants of COCOON 2015 (21st Annual International Computing and Combinatorics Conference). The 6 papers accepted for this special issue were selected using the rigorous external review process of Algorithmica. Included are topics of both theoretical and practical importance, such as algorithmic game theory, graph and network flow theory, approximation algorithms, computational geometry, complexity theory, etc.

We would like to take this opportunity to show our great appreciations to all the people involved in this process in helping us to complete this special issue, including the authors, the reviewers and the journal.

$\triangle$ Dachuan Xu

xudc@bjut.edu.cn

1 Faculty of Business Administration, University of New Brunswick, Fredericton, NB, Canada

2 Department of Applied Mathematics, Beijing University of Technology, Beijing,

People's Republic of China 